\title{
Decomposition of network communication games
}

\author{
Bas Dietzenbacher ${ }^{1}$. Peter Borm ${ }^{1}$. \\ Ruud Hendrickx ${ }^{1}$
}

Received: 9 December 2015 / Accepted: 28 January 2017 / Published online: 15 February 2017 C The Author(s) 2017. This article is published with open access at Springerlink.com

\begin{abstract}
Using network control structures, this paper introduces a general class of network communication games and studies their decomposition into unanimity games. We obtain a relation between the dividends in any network communication game and its underlying transferable utility game, which depends on the structure of the communication network. Moreover, we introduce a new class of network control values which contains both the Myerson value and the position value. The decomposition results are used to explicitly express these values in terms of dividends.
\end{abstract}

Keywords Network control structures · Network communication games - Decomposition theory $\cdot$ Network control values $\cdot$ Myerson value $\cdot$ Position value

Mathematics Subject Classification $05 \mathrm{C} 57 \cdot 91 \mathrm{~A} 12 \cdot 91 \mathrm{~A} 43$

\section{Introduction}

Cooperative game theory analyzes allocations of joint revenues among cooperating players, taking the economic possibilities of subcoalitions into account. To describe an allocation problem for a set of players, Von Neumann and Morgenstern (1944) introduced the model of a transferable utility game, in which a characteristic function assigns to each subgroup of the cooperating players its worth, a number reflecting the

The authors thank two anonymous referees and the associate editor for useful comments and suggestions.

$凶 \quad$ Bas Dietzenbacher

b.j.dietzenbacher@tilburguniversity.edu

1 CentER and Department of Econometrics and Operations Research, Tilburg University, P.O. Box 90153, 5000 LE Tilburg, The Netherlands 
economic possibilities of the coalition if it acts on its own. Shapley (1953) introduced a well-known solution for this model, known as the Shapley value, which divides the dividend of each coalition (cf. Harsanyi 1959) equally among its members.

In a cooperative game with communication structure, the players are subject to cooperation restrictions. Myerson (1977) introduced communication situations in which these cooperation restrictions are modeled by an undirected graph. Vertices of the undirected graph represent the players of the game and there is an edge between two vertices if and only if the corresponding players are able to communicate directly. A coalition can attain its worth if its members are able to communicate, i.e. if their corresponding vertices induce a connected subgraph.

Myerson (1977) introduced the graph-restricted game corresponding to a communication situation in which each coalition of vertices is assigned the sum of the worths of the components in its induced subgraph. We refer to this game as the corresponding vertex game. Owen (1986) studied the decomposition into unanimity games of these vertex games for the special case that the communication network is cycle-free. The Myerson value of a communication situation is defined as the Shapley value of the corresponding vertex game.

Borm et al. (1992) introduced a game on the edges corresponding to a communication situation in which each coalition of edges is assigned the sum of the worths of the components in its induced subgraph. We refer to this game as the corresponding edge game. Borm et al. (1992) also studied the decomposition into unanimity games of these edge games for the special case that the communication network is cycle-free. The position value of a communication situation assigns to each player half of the payoffs allocated to its incident edges by the Shapley value of the corresponding edge game.

This paper introduces a general class of network communication games and a corresponding class of network control values for communication situations. A network communication game is a transferable utility game integrating the features of a communication situation and a network control structure on a communication network. Here, a network control structure models the way in which the vertices and edges of the graph control the communication network. Where Myerson (1977) considered the vertices and Borm et al. (1992) considered the edges as controllers of the network, a network control structure allows both the vertices and edges to control the network in any way. In the corresponding network communication game, each coalition of vertices and edges is assigned the sum of the worths of the components in the subgraph which the members control together.

Focusing on the decomposition into unanimity games of network communication games, it turns out that a communication situation with an underlying unanimity game induces a simple network communication game for any network control structure. The minimal winning coalitions in this game play a central role in its decomposition. We obtain a relation between the dividends in the network communication game and the underlying transferable utility game, which depends on the structure of the communication network. This relation is used to extend the results of Owen (1986) and Borm et al. (1992) for cycle-free networks to all undirected graphs.

For each network control structure, the corresponding network control value of a communication situation assigns to each player the payoff allocated by the Shapley 
value of the corresponding network communication game to its corresponding vertex and half of the payoff allocated to its incident edges. The Myerson value and the position value are network control values which correspond to specific network control structures. We derive an explicit expression of any network control value in terms of the dividends in the underlying transferable utility game.

The main aim of this paper is to develop the decomposition theory for network communication games as a mathematical tool which can be used to derive any network control value for communication situations in a structured way. Future research should study further interpretations and applications of this new framework. Moreover, one could aim to axiomatically characterize the class of all network control values or a specific network control value for communication situations.

This paper is organized in the following way. Section 2 provides an overview of the basic game theoretic and graph theoretic notions and notations. Section 3 formally introduces network control structures, network communication games and network control values, and studies the decomposition into unanimity games. Section 4 discusses the Myerson value and the position value, and the decomposition of their corresponding vertex games and edge games. Section 5 illustrates how the decomposition theory can be extended to more general communication structures such as multigraphs and hypergraphs.

\section{Preliminaries}

Let $N$ be a nonempty and finite set of players. The set of all coalitions is denoted by $2^{N}=\{S \mid S \subseteq N\}$. A collection of coalitions $\mathcal{B} \subseteq 2^{N}$ is called a Sperner family if $R \not \subset S$ for all $R, S \in \mathcal{B}$. A transferable utility game (cf. Von Neumann and Morgenstern 1944) is a pair $(N, v)$ in which $v: 2^{N} \rightarrow \mathbb{R}$ is a characteristic function assigning to each coalition $S \in 2^{N}$ a worth $v(S) \in \mathbb{R}$ such that $v(\emptyset)=0$. The worth of a coalition can be considered as the maximal joint revenue of the members which can be obtained without any assistance of a player which is not a member. Let $\mathrm{TU}^{N}$ denote the class of all transferable utility games with player set $N$. For convenience, we denote a TU-game by $v \in \mathrm{TU}^{N}$. A TU-game $v \in \mathrm{TU}^{N}$ is called simple if the following three conditions are satisfied:

(i) $v(S) \in\{0,1\}$ for all $S \in 2^{N}$;

(ii) $v(N)=1$;

(iii) $v(R) \leq v(S)$ for all $R, S \in 2^{N}$ for which $R \subseteq S$.

Let $\mathrm{SI}^{N}$ denote the class of all simple games with player set $N$. A coalition $S \in 2^{N}$ is called winning in $v \in \mathrm{SI}^{N}$ if $v(S)=1$ and losing if $v(S)=0$. The collection of minimal winning coalitions in $v \in \mathrm{SI}^{N}$ is given by

$$
\mathcal{M}(v)=\left\{S \in 2^{N} \mid v(S)=1, \forall_{R \subset S}: v(R)=0\right\} .
$$

The maximum game $\max \{v \mid v \in \mathcal{V}\} \in \mathrm{TU}^{N}$ of a nonempty and finite set of transferable utility games $\mathcal{V} \subset \mathrm{TU}^{N}$ is defined by $\max \{v \mid v \in \mathcal{V}\}(S)=\max \{v(S) \mid v \in \mathcal{V}\}$ for all $S \in 2^{N}$. The minimum game is defined analogously. Note that both the maximum game and the minimum game of a nonempty set of simple games are simple. 
The unanimity game $u_{R} \in \mathrm{SI}^{N}$ on $R \in 2^{N} \backslash\{\emptyset\}$ is for all $S \in 2^{N}$ defined by

$$
u_{R}(S)= \begin{cases}1 & \text { if } R \subseteq S \\ 0 & \text { if } R \nsubseteq S\end{cases}
$$

We have $v \in \mathrm{SI}^{N}$ and $\mathcal{M}(v)=\mathcal{B}$ if and only if $\mathcal{B} \subseteq 2^{N} \backslash\{\emptyset\}$ is a nonempty Sperner family and $v=\max \left\{u_{R} \mid R \in \mathcal{B}\right\}$.

A TU-game $v \in \mathrm{TU}^{N}$ can be uniquely decomposed into unanimity games,

$$
v=\sum_{S \in 2^{N} \backslash\{\varnothing\}} \Delta^{v}(S) u_{S},
$$

where $\Delta^{v}: 2^{N} \backslash\{\emptyset\} \rightarrow \mathbb{R}$ assigns to each nonempty coalition $S \in 2^{N} \backslash\{\emptyset\}$ its dividend (cf. Harsanyi 1959)

$$
\Delta^{v}(S)=\sum_{R \subseteq S}(-1)^{|S|-|R|} v(R) .
$$

A solution for transferable utility games $f: \mathrm{TU}^{N} \rightarrow \mathbb{R}^{N}$ assigns to any TU-game $v \in \mathrm{TU}^{N}$ a payoff allocation $f(v) \in \mathbb{R}^{N}$ such that $\sum_{i \in N} f_{i}(v)=v(N)$. The Shapley value (cf. Shapley 1953) $\Phi: \mathrm{TU}^{N} \rightarrow \mathbb{R}^{N}$ is for all $v \in \mathrm{TU}^{N}$ and all $i \in N$ given by

$$
\Phi_{i}(v)=\sum_{S \in 2^{N}: i \in S} \frac{1}{|S|} \Delta^{v}(S) .
$$

Let $E \subseteq\left\{S \in 2^{N}|| S \mid=2\right\}$ be a set of unordered pairs of players. The pair $(N, E)$ represents an undirected graph in which $N$ is the set of vertices and $E$ is the set of edges. For all $i \in N$ we denote $E_{i}=\{e \in E \mid i \in e\}$. For all $S \in 2^{N}$ we denote $E[S]=\{e \in E \mid e \subseteq S\}$. For all $T \in 2^{E}$ we denote $N[T]=\left\{i \in N \mid i \in \bigcup_{e \in T} e\right\}$. A pair $(S, T)$ is called a subgraph of $(N, E)$ if $S \in 2^{N}, T \in 2^{E}$ and $N[T] \subseteq S$. The collection of all subgraphs of $(N, E)$ is denoted by $\mathcal{G}^{N, E}$. Let $N[H]$ denote the set of vertices and let $E[H]$ denote the set of edges of a subgraph $H \in \mathcal{G}^{N, E}$, respectively. The subgraph induced by $S \in 2^{N}$ is $(S, E[S])$. The subgraph induced by $T \in 2^{E}$ is $(N[T], T)$.

A path in $(S, T) \in \mathcal{G}^{N, E}$ from $i_{1} \in S$ to $i_{n} \in S$ is a sequence $\left(i_{k}\right)_{k=1}^{n}$ of $n \geq 2$ distinct vertices in $S$ for which $\left\{i_{k}, i_{k+1}\right\} \in T$ for all $k \in\{1, \ldots, n-1\}$. A subgraph $H \in \mathcal{G}^{N, E}$ connects $R \in 2^{N} \backslash\{\emptyset\}$ if for any $i, j \in R, i \neq j$ there exists a path in $H$ from $i$ to $j$. A coalition $C \in 2^{N} \backslash\{\emptyset\}$ is called a component in $H \in \mathcal{G}^{N, E}$ if $H$ connects $C$ and $H$ does not connect any $R \in 2^{N} \backslash\{\emptyset\}$ with $C \subset R$. The collection of all components in $H \in \mathcal{G}^{N, E}$ is denoted by $\mathcal{K}(H)$. A subgraph $(S, T) \in \mathcal{G}^{N, E}$ is called connected if it connects $S$. A connected subgraph $(S, T) \in \mathcal{G}^{N, E}$ is called cycle-free if for any $i, j \in S, i \neq j$ there exists a unique path in $(S, T)$ from $i$ to $j$.

A subgraph $(S, E[S]) \in \mathcal{G}^{N, E}$ is called a minimal $R$-connecting vertex-induced subgraph if it connects $R \in 2^{N} \backslash\{\emptyset\}$ and any $\left(S^{\prime}, E\left[S^{\prime}\right]\right)$ with $S^{\prime} \subset S$ does not connect $R$. The collection of coalitions of vertices which induce a minimal $R$-connecting vertex-induced subgraph is denoted by $\mathcal{N}_{E}^{R} \subseteq 2^{N} \backslash\{\emptyset\}$. A subgraph $(N[T], T) \in \mathcal{G}^{N, E}$ 
is called a minimal $R$-connecting edge-induced subgraph if it connects $R \in 2^{N} \backslash\{\emptyset\}$ and any $\left(N\left[T^{\prime}\right], T^{\prime}\right)$ with $T^{\prime} \subset T$ does not connect $R$. The collection of coalitions of edges which induce a minimal $R$-connecting edge-induced subgraph is denoted by $\mathcal{E}_{N}^{R} \subseteq 2^{E} \backslash\{\emptyset\}$

A communication situation (cf. Myerson 1977) is a triple $(N, v, E)$ in which $v \in \mathrm{TU}^{N}$ is a transferable utility game and $(N, E)$ is an undirected graph representing the communication possibilities between the players. We assume that $v \in \mathrm{TU}^{N}$ is zero-normalized, i.e. $v(\{i\})=0$ for all $i \in N$, and that $(N, E)$ is connected in any communication situation $(N, v, E)$. Let $\mathrm{CS}^{N, E}$ denote the class of all such communication situations with communication network $(N, E)$. For convenience, we denote a communication situation by $v \in \mathrm{CS}^{N, E}$. A solution for communication situations $f: \mathrm{CS}^{N, E} \rightarrow \mathbb{R}^{N}$ assigns to any communication situation $v \in \mathrm{CS}^{N, E}$ a payoff allocation $f(v) \in \mathbb{R}^{N}$ such that $\sum_{i \in N} f_{i}(v)=v(N)$.

The vertex game $w_{E}^{v} \in \mathrm{TU}^{N}$ corresponding to $v \in \mathrm{CS}^{N, E}$ (cf. Myerson 1977) is for all $S \in 2^{N}$ defined by

$$
w_{E}^{v}(S)=\sum_{C \in \mathcal{K}(S, E[S])} v(C) .
$$

The Myerson value $\mu: \mathrm{CS}^{N, E} \rightarrow \mathbb{R}^{N}$ is for all $v \in \mathrm{CS}^{N, E}$ and all $i \in N$ given by

$$
\mu_{i}(v)=\Phi_{i}\left(w_{E}^{v}\right)
$$

The edge game $w_{N}^{v} \in \mathrm{TU}^{E}$ corresponding to $v \in \mathrm{CS}^{N, E}$ (cf. Borm et al. 1992) is for all $T \in 2^{E}$ defined by

$$
w_{N}^{v}(T)=\sum_{C \in \mathcal{K}(N[T], T)} v(C) .
$$

The position value $\pi: \mathrm{CS}^{N, E} \rightarrow \mathbb{R}^{N}$ is for all $v \in \mathrm{CS}^{N, E}$ and all $i \in N$ given by

$$
\pi_{i}(v)=\frac{1}{2} \sum_{e \in E_{i}} \Phi_{e}\left(w_{N}^{v}\right)
$$

\section{Decomposition of network communication games}

In this section we introduce network communication games and study their decomposition into unanimity games. The corresponding network control structure explicitly models the control of the vertices and edges in the underlying communication network.

Definition 3.1 (Network control structure) A network control structure is a triple $(N, E, G)$ in which $(N, E)$ is an undirected graph and $G: 2^{N \cup E} \rightarrow \mathcal{G}^{N, E}$ is a control function assigning to each coalition of vertices and edges a subgraph of $(N, E)$ such that 
(i) $G(\emptyset)=(\emptyset, \varnothing)$;

(ii) $G(N \cup E)=(N, E)$;

(iii) $N[G(Z)] \subseteq N\left[G\left(Z^{\prime}\right)\right]$ and $E[G(Z)] \subseteq E\left[G\left(Z^{\prime}\right)\right]$ for all $Z, Z^{\prime} \in 2^{N \cup E}$ with $Z \subseteq Z^{\prime}$.

Let $\operatorname{NCS}^{N, E}$ denote the class of all network control structures on $(N, E)$. For convenience, we denote a network control structure by $G \in \operatorname{NCS}^{N, E}$.

Example 1 Let $N=\{1,2,3\}$, let $E=\{\{1,2\},\{2,3\}\}$ and let $G \in \operatorname{NCS}^{N, E}$ be the network control structure with $G(Z)=((Z \cap N) \cup N[Z \cap E],(Z \cap E) \cup E[Z \cap N])^{1}$ for all $Z \in 2^{N \cup E}$. This means that each vertex is controlled by itself and its incident edges, and each edge is controlled by itself and its two endpoints together. The graph $(N, E)$ is depicted below.

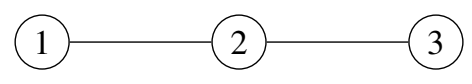

We have

$$
\begin{aligned}
G(\{1,2\}) & =G(\{\{1,2\}\})=G(\{1,\{1,2\}\})=G(\{1,2,\{1,2\}\})=(\{1,2\},\{\{1,2\}\}) ; \\
G(\{1,3\}) & =(\{1,3\}, \emptyset) ; \\
G(\{3,\{1,2\}\}) & =G(\{1,3,\{1,2\}\})=(\{1,2,3\},\{\{1,2\}\}) \\
G(N) & =G(E)=G(N \cup E)=(N, E) .
\end{aligned}
$$

A network communication game combines a network control structure $G \in$ $\mathrm{NCS}^{N, E}$ and a communication situation $v \in \mathrm{CS}^{N, E}$ into a transferable utility game on $N \cup E$ in which the worth of a coalition of vertices and edges equals the sum of the worths of the components in the subgraph which the members control together.

Definition 3.2 (Network communication game) Let $G \in \mathrm{NCS}^{N, E}$ be a network control structure and let $v \in \mathrm{CS}^{N, E}$ be a communication situation. In the corresponding network communication game $w_{G}^{v} \in \mathrm{TU}^{N \cup E}$ the worth of each coalition of vertices and edges $Z \in 2^{N \cup E}$ is given by

$$
w_{G}^{v}(Z)=\sum_{C \in \mathcal{K}(G(Z))} v(C) .
$$

For any network control structure, the network control value of a communication situation assigns to each player the payoff allocated by the Shapley value of the corresponding network communication game to its corresponding vertex and half of the payoff allocated to its incident edges.

\footnotetext{
1 The elaborate notation of this network control structure is needed to ensure that each coalition of vertices and edges is assigned a subgraph of the communication network.
} 
Definition 3.3 (Network control value) Let $G \in \mathrm{NCS}^{N, E}$ be a network control structure. The corresponding network control value $\phi^{G}: \mathrm{CS}^{N, E} \rightarrow \mathbb{R}^{N}$ is for any communication situation $v \in \mathrm{CS}^{N, E}$ and any player $i \in N$ given by

$$
\phi_{i}^{G}(v)=\Phi_{i}\left(w_{G}^{v}\right)+\frac{1}{2} \sum_{e \in E_{i}} \Phi_{e}\left(w_{G}^{v}\right) .
$$

For any $G \in \mathrm{NCS}^{N, E}$, let $\mathcal{M}_{G}^{R} \subseteq 2^{N \cup E} \backslash\{\emptyset\}$ denote the collection of coalitions of vertices and edges $Z \in 2^{N \cup E}$ for which $G(Z)$ connects $R \in 2^{N} \backslash\{\emptyset\}$ and any $G\left(Z^{\prime}\right)$ with $Z^{\prime} \subset Z$ does not connect $R$. For any network control structure, it turns out that a communication situation with an underlying unanimity game corresponds to a simple network communication game with this collection of minimal winning coalitions.

Lemma 3.1 Let $G \in \operatorname{NCS}^{N, E}$ and let $R \in 2^{N} \backslash\{\emptyset\}$. Then $w_{G}^{u_{R}} \in \mathrm{SI}^{N \cup E}$ and $\mathcal{M}\left(w_{G}^{u_{R}}\right)=\mathcal{M}_{G}^{R}$.

Proof Since for any coalition of vertices and edges $Z \in 2^{N \cup E}$ there is at most one component $C \in \mathcal{K}(G(Z))$ for which $R \subseteq C$, we can write for each $Z \in 2^{N \cup E}$

$$
\begin{aligned}
w_{G}^{u_{R}}(Z) & \stackrel{(5)}{=} \sum_{C \in \mathcal{K}(G(Z))} u_{R}(C)=|\{C \in \mathcal{K}(G(Z)) \mid R \subseteq C\}| \\
& =\left\{\begin{array}{ll}
1 & \text { if } \exists_{C \in \mathcal{K}(G(Z))}: R \subseteq C ; \\
0 & \text { if } \forall_{C \in \mathcal{K}(G(Z))}: R \nsubseteq C
\end{array}= \begin{cases}1 & \text { if } G(Z) \text { connects } R ; \\
0 & \text { if } G(Z) \text { does not connect } R .\end{cases} \right.
\end{aligned}
$$

Since $(N, E)$ is connected, $G(N \cup E)=(N, E)$ connects $R$, so $w_{G}^{u_{R}}(N \cup E)=1$. If $G(Z)$ connects $R$ for some $Z \in 2^{N \cup E}$, then $G\left(Z^{\prime}\right)$ connects $R$ for all $Z^{\prime} \in 2^{N \cup E}$ for which $Z \subseteq Z^{\prime}$, so $w_{G}^{u_{R}}(Z) \leq w_{G}^{u_{R}}\left(Z^{\prime}\right)$ for all $Z, Z^{\prime} \in 2^{N \cup E}$ for which $Z \subseteq Z^{\prime}$. This means that $w_{G}^{u_{R}}(Z) \in\{0,1\}$ for all $Z \in 2^{N \cup E}, w_{G}^{u_{R}}(N \cup E)=1$ and $w_{G}^{u_{R}}(Z) \leq$ $w_{G}^{u_{R}}\left(Z^{\prime}\right)$ for all $Z, Z^{\prime} \in 2^{N \cup E}$ for which $Z \subseteq Z^{\prime}$. Hence, $w_{G}^{u_{R}} \in \mathrm{SI}^{N \cup E}$. Moreover, $\mathcal{M}\left(w_{G}^{u_{R}}\right)=\mathcal{M}_{G}^{R}$ is a direct consequence of Eq. (1).

Lemma 3.2 Let $v \in \mathrm{SI}^{N}$. Then

$$
v=\sum_{\mathcal{B} \subseteq \mathcal{M}(v): \mathcal{B} \neq \emptyset}(-1)^{|\mathcal{B}|+1} u_{\left(\bigcup_{R \in \mathcal{B}} R\right)} .
$$

Moreover, for each $S \in 2^{N} \backslash\{\emptyset\}$ we have

$$
\Delta^{v}(S)=\sum_{\mathcal{B} \subseteq \mathcal{M}(v): \bigcup_{R \in \mathcal{B}} R=S}(-1)^{|\mathcal{B}|+1} .
$$

Proof Since Eq. (8) is a direct consequence of Eq. (7), it suffices to show Eq. (7). We first show that for each $R^{\prime} \in 2^{N} \backslash\{\emptyset\}$ we have

$$
\min \left\{v, u_{R^{\prime}}\right\}=\sum_{R \in 2^{N} \backslash\{\emptyset\}} \Delta^{v}(R) u_{R \cup R^{\prime}}
$$


We know $v=\sum_{R \in 2^{N} \backslash\{\emptyset\}} \Delta^{v}(R) u_{R}$. Let $S \in 2^{N}$. Then $v(S) \in\{0,1\}$. Let $R^{\prime} \in$ $2^{N} \backslash\{\emptyset\}$ and suppose that we have $R^{\prime} \nsubseteq S$. Then we have $u_{R^{\prime}}(S)=0$ and $R \cup R^{\prime} \nsubseteq S$ for any $R \in 2^{N} \backslash\{\emptyset\}$, which implies that $u_{R \cup R^{\prime}}(S)=0$ for any $R \in 2^{N} \backslash\{\emptyset\}$. Consequently,

$\min \left\{v, u_{R^{\prime}}\right\}(S)=\min \left\{v(S), u_{R^{\prime}}(S)\right\}=\min \{v(S), 0\}=0=\sum_{R \in 2^{N} \backslash\{\emptyset\}} \Delta^{v}(R) u_{R \cup R^{\prime}}(S)$.

Next, suppose that we have $R^{\prime} \subseteq S$. Then we have $u_{R^{\prime}}(S)=1$, and $R \cup R^{\prime} \subseteq S$ if and only if $R \subseteq S$ for any $R \in 2^{N} \backslash\{\emptyset\}$, which implies that $u_{R \cup R^{\prime}}(S)=u_{R}(S)$ for any $R \in 2^{N} \backslash\{\emptyset\}$. Consequently,

$$
\begin{aligned}
\min \left\{v, u_{R^{\prime}}\right\}(S) & =\min \left\{v(S), u_{R^{\prime}}(S)\right\}=\min \{v(S), 1\}=v(S) \\
& =\sum_{R \in 2^{N} \backslash\{\emptyset\}} \Delta^{v}(R) u_{R}(S)=\sum_{R \in 2^{N} \backslash\{\emptyset\}} \Delta^{v}(R) u_{R \cup R^{\prime}}(S) .
\end{aligned}
$$

Hence, Eq. (9) holds.

Next, we prove Eq. (7) by induction on $|\mathcal{M}(v)|$. Suppose that we have $|\mathcal{M}(v)|=1$ and denote $\mathcal{M}(v)=\left\{R_{1}\right\}$. Then we can write $v=\max \left\{u_{R} \mid R \in \mathcal{M}(v)\right\}=\max \left\{u_{R_{1}}\right\}=u_{R_{1}}=\sum_{\mathcal{B} \subseteq \mathcal{M}(v): \mathcal{B} \neq \emptyset}(-1)^{|\mathcal{B}|+1} u_{\left(\bigcup_{R \in \mathcal{B}} R\right)}$.

Let $n \in \mathbb{N}$ and assume that for any simple game $v^{\prime} \in \mathrm{SI}^{N}$ for which $\left|\mathcal{M}\left(v^{\prime}\right)\right|=n$ we have $v^{\prime}=\sum_{\mathcal{B} \subseteq \mathcal{M}\left(v^{\prime}\right): \mathcal{B} \neq \emptyset}(-1)^{|\mathcal{B}|+1} u_{\left(\bigcup_{R \in \mathcal{B}} R\right)}$. Suppose that we have $|\mathcal{M}(v)|=n+1$. Denote $\mathcal{M}(v)=\left\{R_{1}, \ldots, R_{n+1}\right\}$. Then we can write

$$
\begin{aligned}
v & =\max \left\{u_{R} \mid R \in \mathcal{M}(v)\right\} \\
& =\max \left\{u_{R_{1}}, \ldots, u_{R_{n+1}}\right\} \\
& =\max \left\{\max \left\{u_{R_{1}}, \ldots, u_{R_{n}}\right\}, u_{R_{n+1}}\right\} \\
& =\max \left\{u_{R_{1}}, \ldots, u_{R_{n}}\right\}+u_{R_{n+1}}-\min \left\{\max \left\{u_{R_{1}}, \ldots, u_{R_{n}}\right\}, u_{R_{n+1}}\right\} \\
& \stackrel{(9)}{=} \sum_{\mathcal{B} \subseteq\left\{R_{1}, \ldots, R_{n}\right\}: \mathcal{B} \neq \emptyset}(-1)^{|\mathcal{B}|+1} u_{\left(\bigcup_{R \in \mathcal{B}} R\right)}+u_{R_{n+1}}-\sum_{\mathcal{B} \subseteq\left\{R_{1}, \ldots, R_{n}\right\}: \mathcal{B} \neq \emptyset}(-1)^{|\mathcal{B}|+1} u_{\left(\cup_{R \in \mathcal{B}} R\right) \cup R_{n+1}}(-1)^{|\mathcal{B}|+1} u_{\left(\cup_{R \in \mathcal{B}} R\right)} \\
= & \sum_{\mathcal{B} \subseteq\left\{R_{1}, \ldots, R_{n+1}\right\}: \mathcal{B} \neq \emptyset}(-1)^{|\mathcal{B}|+1} u_{\left(\bigcup_{R \in \mathcal{B}} R\right) .} \\
= & \sum_{\mathcal{B} \subseteq \mathcal{M}(v): \mathcal{B} \neq \emptyset}
\end{aligned}
$$

Example 2 Let $N=\{1,2,3\}$, let $E=\{\{1,2\},\{2,3\}\}$ and let $G \in \operatorname{NCS}^{N, E}$ be the network control structure with $G(Z)=((Z \cap N) \cup N[Z \cap E],(Z \cap E) \cup E[Z \cap N])$ for all $Z \in 2^{N \cup E}$. This means that each vertex is controlled by itself and its incident edges, and each edge is controlled by itself and its two endpoints together as in Example 1. We have

$$
\mathcal{M}_{G}^{\{1,3\}}=\{\{1,2,3\},\{1,2,\{2,3\}\},\{2,3,\{1,2\}\},\{\{1,2\},\{2,3\}\}\} .
$$


Note that, although $(N, E)$ is cycle-free, $\mathcal{M}_{G}^{\{1,3\}}$ contains multiple elements. Consider the communication situation $u_{\{1,3\}} \in \mathrm{CS}^{N, E}$. Using Lemmas 3.1 and 3.2, we can write

$$
\begin{aligned}
& w_{G}^{u_{\{1,3\}}}=u_{\{1,2,3\}}+u_{\{1,2,\{2,3\}\}}+u_{\{2,3,\{1,2\}\}}+u_{\{\{1,2\},\{2,3\}\}} \\
& -u_{\{1,2,3,\{2,3\}\}}-u_{\{1,2,3,\{1,2\}\}}-u_{\{1,2,3,\{1,2\},\{2,3\}\}} \\
& -u_{\{1,2,3,\{1,2\},\{2,3\}\}}-u_{\{1,2,\{1,2\},\{2,3\}\}}-u_{\{2,3,\{1,2\},\{2,3\}\}} \\
& +u_{\{1,2,3,\{1,2\},\{2,3\}\}}+u_{\{1,2,3,\{1,2\},\{2,3\}\}}+u_{\{1,2,3,\{1,2\},\{2,3\}\}}+u_{\{1,2,3,\{1,2\},\{2,3\}\}} \\
& -u_{\{1,2,3,\{1,2\},\{2,3\}\}} \\
& =u_{\{1,2,3\}}+u_{\{1,2,\{2,3\}\}}+u_{\{2,3,\{1,2\}\}}+u_{\{\{1,2\},\{2,3\}\}}-u_{\{1,2,3,\{1,2\}\}}-u_{\{1,2,3,\{2,3\}\}} \\
& -u_{\{1,2,\{1,2\},\{2,3\}\}}-u_{\{2,3,\{1,2\},\{2,3\}\}}+u_{\{1,2,3,\{1,2\},\{2,3\}\}} \text {. }
\end{aligned}
$$

The corresponding network control value is given by

$$
\phi^{G}\left(u_{\{1,3\}}\right)=\left(\frac{31}{120}, \frac{58}{120}, \frac{31}{120}\right) .
$$

For any network control structure, the dividends in general network communication games can be derived from the dividends in the underlying transferable utility game and the dividends in network communication games with an underlying unanimity game.

Lemma 3.3 Let $G \in \mathrm{NCS}^{N, E}$, let $v \in \mathrm{CS}^{N, E}$ and let $Z \in 2^{N \cup E} \backslash\{\emptyset\}$. Then

$$
\Delta^{w_{G}^{v}}(Z)=\sum_{R \in 2^{N} \backslash\{\emptyset\}} \Delta^{v}(R) \Delta^{w_{G}^{u_{R}}}(Z) .
$$

Proof We can write

$$
\begin{aligned}
\Delta^{w_{G}^{v}}(Z) & \stackrel{(3)}{=} \sum_{Z^{\prime} \subseteq Z}(-1)^{|Z|-\left|Z^{\prime}\right|} w_{G}^{v}\left(Z^{\prime}\right) \\
& \stackrel{(5)}{=} \sum_{Z^{\prime} \subseteq Z}(-1)^{|Z|-\left|Z^{\prime}\right|} \sum_{C \in \mathcal{K}\left(G\left(Z^{\prime}\right)\right)} v(C) \\
& \stackrel{(2)}{=} \sum_{Z^{\prime} \subseteq Z}(-1)^{|Z|-\left|Z^{\prime}\right|} \sum_{C \in \mathcal{K}\left(G\left(Z^{\prime}\right)\right)}\left(\sum_{R \in 2^{N} \backslash\{\emptyset\}} \Delta^{v}(R) u_{R}(C)\right) \\
& =\sum_{R \in 2^{N} \backslash\{\emptyset\}} \Delta^{v}(R) \sum_{Z^{\prime} \subseteq Z}(-1)^{|Z|-\left|Z^{\prime}\right|} \sum_{C \in \mathcal{K}\left(G\left(Z^{\prime}\right)\right)} u_{R}(C) \\
& \stackrel{(5)}{=} \sum_{R \in 2^{N} \backslash\{\emptyset\}} \Delta^{v}(R) \sum_{Z^{\prime} \subseteq Z}(-1)^{|Z|-\left|Z^{\prime}\right|} w_{G}^{u_{R}}\left(Z^{\prime}\right) \\
& \stackrel{(3)}{=} \sum_{R \in 2^{N} \backslash\{\emptyset\}} \Delta^{v}(R) \Delta_{G}^{w_{R}^{u}}(Z) .
\end{aligned}
$$


Using Lemma 3.3, we can extend the decomposition results for network communication games with an underlying unanimity game to general network communication games for any network control structure and derive an explicit expression of any network control value in terms of the dividends in the underlying transferable utility game.

Theorem 3.4 Let $G \in \mathrm{NCS}^{N, E}$ be a network control structure and let $v \in \mathrm{CS}^{N, E}$ be a communication situation. Then

$$
w_{G}^{v}=\sum_{R \in 2^{N} \backslash\{\emptyset\}} \Delta^{v}(R) \sum_{\mathcal{B} \subseteq \mathcal{M}_{G}^{R}: \mathcal{B} \neq \emptyset}(-1)^{|\mathcal{B}|+1} u_{\left(\bigcup_{Z \in \mathcal{B}} Z\right)}
$$

Proof Using Lemmas 3.1, 3.2 and 3.3, we can write

$$
\begin{aligned}
& w_{G}^{v} \stackrel{(2)}{=} \sum_{Z \in 2^{N \cup E} \backslash\{\emptyset\}} \Delta^{w_{G}^{v}}(Z) u_{Z} \\
& \stackrel{(10)}{=} \sum_{Z \in 2^{N \cup E} \backslash\{\emptyset\}}\left(\sum_{R \in 2^{N} \backslash\{\emptyset\}} \Delta^{v}(R) \Delta^{w_{G}^{u_{R}}}(Z) u_{Z}\right) \\
& =\sum_{R \in 2^{N} \backslash\{\emptyset\}} \Delta^{v}(R) \sum_{Z \in 2^{N \cup E} \backslash\{\emptyset\}} \Delta^{w_{G}^{u_{R}}}(Z) u_{Z} \\
& \stackrel{(2)}{=} \sum_{R \in 2^{N} \backslash\{\emptyset\}} \Delta^{v}(R) w_{G}^{u_{R}} \\
& \stackrel{(7)}{=} \sum_{R \in 2^{N} \backslash\{\emptyset\}} \Delta^{v}(R) \sum_{\mathcal{B} \subseteq \mathcal{M}_{G}^{R}: \mathcal{B} \neq \emptyset}(-1)^{|\mathcal{B}|+1} u_{\left(\bigcup_{Z \in \mathcal{B}} Z\right)} \text {. }
\end{aligned}
$$

Theorem 3.5 Let $G \in \mathrm{NCS}^{N, E}$ be a network control structure, let $v \in \mathrm{CS}^{N, E}$ be a communication situation and let $i \in N$ be a player. Then

$$
\phi_{i}^{G}(v)=\sum_{Z \in 2^{N \cup E}} \frac{|Z \cap\{i\}|+\frac{1}{2}\left|Z \cap E_{i}\right|}{|Z|} \sum_{R \in 2^{N} \backslash\{\emptyset\}} \Delta^{v}(R) \sum_{\mathcal{B} \subseteq \mathcal{M}_{G}^{R}: \bigcup_{Z^{\prime} \in \mathcal{B}} Z^{\prime}=Z}(-1)^{|\mathcal{B}|+1} .
$$


Proof Using Lemmas 3.1, 3.2 and 3.3, we can write

$$
\begin{aligned}
\phi_{i}^{G}(v) & \stackrel{(6)}{=} \Phi_{i}\left(w_{G}^{v}\right)+\frac{1}{2} \sum_{e \in E_{i}} \Phi_{e}\left(w_{G}^{v}\right) \\
& \stackrel{(4)}{=} \sum_{Z \in 2^{N \cup E}: i \in Z} \frac{1}{|Z|} \Delta^{w_{G}^{v}}(Z)+\frac{1}{2} \sum_{e \in E_{i}} \sum_{Z \in 2^{N \cup E}: e \in Z} \frac{1}{|Z|} \Delta^{w_{G}^{v}(Z)} \\
& =\sum_{Z \in 2^{N} \cup E} \frac{|Z \cap\{i\}|+\frac{1}{2}\left|Z \cap E_{i}\right|}{|Z|} \Delta_{G}^{w_{G}^{v}}(Z) \\
& \stackrel{(10)}{=} \sum_{Z \in 2^{N \cup E}} \frac{|Z \cap\{i\}|+\frac{1}{2}\left|Z \cap E_{i}\right|}{|Z|} \sum_{R \in 2^{N} \backslash\{\emptyset\}} \Delta^{v}(R) \Delta^{w_{G}^{u}}(Z) \\
& \stackrel{(8)}{=} \sum_{Z \in 2^{N \cup E}} \frac{|Z \cap\{i\}|+\frac{1}{2}\left|Z \cap E_{i}\right|}{|Z|} \sum_{R \in 2^{N} \backslash\{\emptyset\}} \Delta^{v}(R) \quad \mathcal{B} \subseteq \mathcal{M}_{G}^{R}: \bigcup_{Z^{\prime} \in \mathcal{B}} Z^{\prime}=Z
\end{aligned}
$$

\section{Network control values}

In this section we discuss the Myerson value and the position value, and the decomposition into unanimity games of their corresponding vertex games and edge games. Moreover, we focus on the special case that the underlying communication network is cycle-free.

From the viewpoint of Myerson (1977) the vertices of the graph control the network such that each vertex controls itself and each edge is controlled by its two endpoints together. In other words, each coalition of vertices controls its induced subgraph. This can be described by the network control structure $G \in \operatorname{NCS}^{N, E}$ with $G(Z)=$ $(Z \cap N, E[Z \cap N])$ for all $Z \in 2^{N \cup E}$. We have $\mathcal{M}_{G}^{R}=\mathcal{M}\left(w_{G}^{u_{R}}\right)=\mathcal{M}\left(w_{E}^{u_{R}}\right)=\mathcal{N}_{E}^{R}$ for any $R \in 2^{N} \backslash\{\varnothing\}$ and the corresponding network control value for communication situations coincides with the Myerson value, i.e. $\phi^{G}=\mu$.

From the viewpoint of Borm et al. (1992) the edges of the graph control the network such that each edge controls itself and its both endpoints. In other words, each coalition of edges controls its induced subgraph. This can be described by the network control structure $G \in \mathrm{NCS}^{N, E}$ with $G(Z)=(N[Z \cap E], Z \cap E)$ for all $Z \in 2^{N \cup E}$. We have $\mathcal{M}_{G}^{R}=\mathcal{M}\left(w_{G}^{u_{R}}\right)=\mathcal{M}\left(w_{N}^{u_{R}}\right)=\mathcal{E}_{N}^{R}$ for any $R \in 2^{N} \backslash\{\emptyset\}$ and the corresponding network control value for communication situations coincides with the position value, i.e. $\phi^{G}=\pi$.

Using Theorem 3.4, we find the decomposition into unanimity games of vertex games and edge games in terms of the dividends in the transferable utility game underlying the corresponding communication situation. 
Theorem 4.1 Let $v \in \mathrm{CS}^{N, E}$ be a communication situation. Then

$$
\begin{aligned}
w_{E}^{v} & =\sum_{R \in 2^{N} \backslash\{\emptyset\}} \Delta^{v}(R) \sum_{\mathcal{B} \subseteq \mathcal{N}_{E}^{R}: \mathcal{B} \neq \emptyset}(-1)^{|\mathcal{B}|+1} u_{\left(\bigcup_{S \in \mathcal{B}} S\right)} \\
\text { and } w_{N}^{v} & =\sum_{R \in 2^{N} \backslash\{\emptyset\}} \Delta^{v}(R) \sum_{\mathcal{B} \subseteq \mathcal{E}_{N}^{R}: \mathcal{B} \neq \emptyset}(-1)^{|\mathcal{B}|+1} u_{\left(\bigcup_{T \in \mathcal{B}} T\right)} .
\end{aligned}
$$

Using Theorem 3.5, we obtain new expressions of the Myerson value and the position value in terms of the dividends of the transferable utility game underlying the corresponding communication situation.

Theorem 4.2 Let $v \in \mathrm{CS}^{N, E}$ be a communication situation and let $i \in N$ be a player. Then

$$
\begin{aligned}
\mu_{i}(v) & =\sum_{S \in 2^{N}: i \in S} \frac{1}{|S|} \sum_{R \in 2^{N} \backslash\{\emptyset\}} \Delta^{v}(R) \sum_{\mathcal{B} \subseteq \mathcal{N}_{E}^{R}: \bigcup_{S^{\prime} \in \mathcal{B}} S^{\prime}=S}(-1)^{|\mathcal{B}|+1} \\
\text { and } \pi_{i}(v) & =\sum_{T \in 2^{E}} \frac{\left|T \cap E_{i}\right|}{2|T|} \sum_{R \in 2^{N} \backslash\{\emptyset\}} \Delta^{v}(R) \sum_{\mathcal{B} \subseteq \mathcal{E}_{N}^{R}: \bigcup_{T^{\prime} \in \mathcal{B}} T^{\prime}=T}(-1)^{|\mathcal{B}|+1} .
\end{aligned}
$$

If the underlying communication network is cycle-free, it contains a unique minimal $R$-connecting vertex-induced subgraph and a unique minimal $R$-connecting edgeinduced subgraph which both coincide for any $R \in 2^{N}$ with $|R| \geq 2$. This means that any vertex game or edge game for which a unanimity game underlies the corresponding communication situation is a unanimity game as well.

Example 3 Let $N=\{1,2,3\}$ and let $E=\{\{1,2\},\{2,3\}\}$ as in Example 1 and Example 2. The graph $(N, E)$ is cycle-free. We have $\mathcal{N}_{E}^{\{1,3\}}=\{N\}$ and $\mathcal{E}_{N}^{\{1,3\}}=\{E\}$. Using Theorem 4.1, we can write

$$
w_{E}^{u_{\{1,3\}}}=u_{N} \quad \text { and } \quad w_{N}^{u\{1,3\}}=u_{E}
$$

Using Theorem 4.2, we derive

$$
\mu\left(u_{\{1,3\}}\right)=\left(\frac{1}{3}, \frac{1}{3}, \frac{1}{3}\right) \text { and } \pi\left(u_{\{1,3\}}\right)=\left(\frac{1}{4}, \frac{1}{2}, \frac{1}{4}\right)
$$

If $(N, E)$ is cycle-free, let $S_{E}^{R} \in 2^{N} \backslash\{\emptyset\}$ denote for any $R \in 2^{N}$ with $|R| \geq 2$ the unique coalition of vertices for which $S_{E}^{R} \in \mathcal{N}_{E}^{R}$. We have $\mathcal{N}_{E}^{R}=\left\{S_{E}^{R}\right\}$ and $\mathcal{E}_{N}^{R}=$ $\left\{E\left[S_{E}^{R}\right]\right\}$. Moreover, $w_{E}^{u_{R}}=u_{S_{E}^{R}}$ and $w_{N}^{u_{R}}=u_{E\left[S_{E}^{R}\right]}$. Combining these observations with Lemma 3.3, we obtain the following relations. 
Corollary 4.3 Let $v \in \mathrm{CS}^{N, E}$ be a communication situation. If $(N, E)$ is cycle-free, then

$$
\begin{aligned}
\Delta^{w_{E}^{v}}(S) & =\sum_{R \in 2^{N} \backslash\{\emptyset\}: S_{E}^{R}=S} \Delta^{v}(R) \text { for all } S \in 2^{N} \backslash\{\emptyset\} \\
\text { and } \Delta^{w_{N}^{v}}(T) & =\sum_{R \in 2^{N} \backslash\{\emptyset\}: E\left[S_{E}^{R}\right]=T} \Delta^{v}(R) \text { for all } T \in 2^{E} \backslash\{\emptyset\} .
\end{aligned}
$$

Corollary 4.3 offers results which were also found by Owen (1986) and Borm et al. (1992). The following results are derived from Theorems 4.1 and 4.2, respectively.

Corollary 4.4 Let $v \in \mathrm{CS}^{N, E}$ be a communication situation. If $(N, E)$ is cycle-free, then

$$
\begin{aligned}
w_{E}^{v} & =\sum_{R \in 2^{N} \backslash\{\emptyset\}} \Delta^{v}(R) u_{S_{E}^{R}} \\
\text { and } w_{N}^{v} & =\sum_{R \in 2^{N} \backslash\{\emptyset\}} \Delta^{v}(R) u_{E\left[S_{E}^{R}\right]} .
\end{aligned}
$$

Corollary 4.5 Let $v \in \mathrm{CS}^{N, E}$ be a communication situation and let $i \in N$ be a player. If $(N, E)$ is cycle-free, then

$$
\begin{aligned}
\mu_{i}(v) & =\sum_{R \in 2^{N} \backslash\{\emptyset\}: i \in S_{E}^{R}} \frac{1}{\left|S_{E}^{R}\right|} \Delta^{v}(R) \\
\text { and } \pi_{i}(v) & =\sum_{R \in 2^{N} \backslash\{\emptyset\}} \frac{\left|E\left[S_{E}^{R}\right] \cap E_{i}\right|}{2\left|E\left[S_{E}^{R}\right]\right|} \Delta^{v}(R) .
\end{aligned}
$$

Example 4 Let $N=\{1,2,3,4\}$ and let $E=\{\{1,2\},\{2,3\},\{2,4\}\}$. The cycle-free graph $(N, E)$ is depicted below.

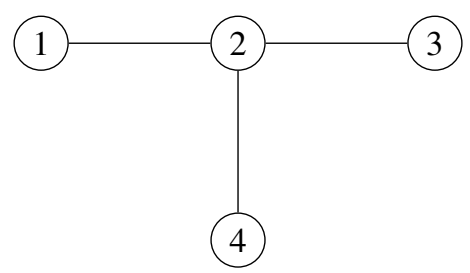

Consider the communication situation $v \in \mathrm{CS}^{N, E}$ for which

$$
v=2 u_{\{1,2\}}+3 u_{\{1,3\}}-3 u_{\{1,2,3\}}+5 u_{\{1,3,4\}}+7 u_{\{1,2,3,4\}} .
$$


Using Corollary 4.4, we can write

$$
\begin{aligned}
w_{E}^{v} & =2 u_{\{1,2\}}+3 u_{\{1,2,3\}}-3 u_{\{1,2,3\}}+5 u_{\{1,2,3,4\}}+7 u_{\{1,2,3,4\}} \\
& =2 u_{\{1,2\}}+12 u_{\{1,2,3,4\}}
\end{aligned}
$$

and

$$
\begin{aligned}
w_{N}^{v} & =2 u_{\{\{1,2\}\}}+3 u_{\{\{1,2\},\{2,3\}\}}-3 u_{\{\{1,2\},\{2,3\}\}}+5 u_{\{\{1,2\},\{2,3\},\{2,4\}\}}+7 u_{\{\{1,2\},\{2,3\},\{2,4\}\}} \\
& =2 u_{\{\{1,2\}\}}+12 u_{\{\{1,2\},\{2,3\},\{2,4\}\}}
\end{aligned}
$$

Using Corollary 4.5, we derive

$$
\begin{array}{rll}
\mu(v) & =(1+3,1+3,3,3)=(4,4,3,3) \\
\text { and } \pi(v) & =(1+2,1+6,2,2)=(3,7,2,2) .
\end{array}
$$

The special uniqueness relation in cycle-free communication networks not only holds for the Myerson value and the position value, but also for other network control values with a specific type of network control structure. In particular, for the network control structure $\widetilde{G} \in \mathrm{NCS}^{N, E}$ with $\widetilde{G}(Z)=(Z \cap N[Z \cap E], Z \cap E[Z \cap N])$ in which each vertex and each edge only controls itself. We have $\mathcal{M}_{\widetilde{G}}^{R}=\left\{N[T] \cup T \mid T \in \mathcal{E}_{N}^{R}\right\}$ for any $R \in 2^{N}$ with $|R| \geq 2$. If $(N, E)$ is cycle-free, we have $\mathcal{M}_{\widetilde{G}}^{R}=\left\{S_{E}^{R} \cup E\left[S_{E}^{R}\right]\right\}$ and $w_{\widetilde{G}}^{u_{R}}=u_{S_{E}^{R} \cup E\left[S_{E}^{R}\right]}$ for any $R \in 2^{N}$ with $|R| \geq 2$.

Example 5 Let $N=\{1,2,3\}$ and let $E=\{\{1,2\},\{2,3\}\}$ as in Example 1, Example 2 and Example 3. The graph $(N, E)$ is cycle-free and we have $S_{E}^{\{1,3\}}=N$. We can write

$$
w_{\widetilde{G}}^{u_{\{1,3\}}}=u_{N \cup E} \quad \text { and } \quad \phi^{\widetilde{G}}\left(u_{\{1,3\}}\right)=\left(\frac{3}{10}, \frac{4}{10}, \frac{3}{10}\right) .
$$

Note that $\phi^{\widetilde{G}}\left(u_{\{1,3\}}\right)=\frac{3}{5} \mu\left(u_{\{1,3\}}\right)+\frac{2}{5} \pi\left(u_{\{1,3\}}\right)$.

In Example 5 we observe that the value $\phi^{\widetilde{G}}$ is a specific convex combination of the Myerson value $\mu$ and the position value $\pi$. This holds for any communication situation with an underlying unanimity game and a cycle-free communication network.

Theorem 4.6 Let $R \in 2^{N}$ with $|R| \geq 2$. If $(N, E)$ is cycle-free, then

$$
\phi^{\widetilde{G}}\left(u_{R}\right)=\frac{\left|S_{E}^{R}\right|}{2\left|S_{E}^{R}\right|-1} \mu\left(u_{R}\right)+\frac{\left|S_{E}^{R}\right|-1}{2\left|S_{E}^{R}\right|-1} \pi\left(u_{R}\right) .
$$


Proof Assume that $(N, E)$ is cycle-free and let $i \in N$. If $i \notin S_{E}^{R}$, then $e \notin E\left[S_{E}^{R}\right]$ for all $e \in E_{i}$, so $\phi_{i}^{\widetilde{G}}\left(u_{R}\right)=\mu_{i}\left(u_{R}\right)=\pi_{i}\left(u_{R}\right)=0$ and the statement follows. Suppose that $i \in S_{E}^{R}$. Using Corollary 4.5 and $\left|E\left[S_{E}^{R}\right]\right|=\left|S_{E}^{R}\right|-1$, we can write

$$
\begin{aligned}
\phi_{i}^{\widetilde{G}}\left(u_{R}\right) & =\Phi_{i}\left(w_{\widetilde{G}}^{u_{R}}\right)+\frac{1}{2} \sum_{e \in E_{i}} \Phi_{e}\left(w_{\widetilde{G}}^{u_{R}}\right) \\
& \stackrel{(4)}{=} \sum_{Z \in 2^{N \cup E}: i \in Z} \frac{1}{|Z|} \Delta^{w_{\widetilde{G}}^{u_{R}}}(Z)+\frac{1}{2} \sum_{e \in E_{i}} \sum_{Z \in 2^{N \cup E}: e \in Z} \frac{1}{|Z|} \Delta^{w_{\widetilde{G}}^{u_{R}}(Z)} \\
& =\frac{1}{\left|S_{E}^{R} \cup E\left[S_{E}^{R}\right]\right|}+\frac{\left|E\left[S_{E}^{R}\right] \cap E_{i}\right|}{2\left|S_{E}^{R} \cup E\left[S_{E}^{R}\right]\right|} \\
& =\frac{1}{2\left|S_{E}^{R}\right|-1}+\frac{\left|E\left[S_{E}^{R}\right] \cap E_{i}\right|}{4\left|S_{E}^{R}\right|-2} \\
& =\frac{\left|S_{E}^{R}\right|}{2\left|S_{E}^{R}\right|-1}\left(\frac{1}{\left|S_{E}^{R}\right|}\right)+\frac{\left|S_{E}^{R}\right|-1}{2\left|S_{E}^{R}\right|-1}\left(\frac{\left|E\left[S_{E}^{R}\right] \cap E_{i}\right|}{2\left|E\left[S_{E}^{R}\right]\right|}\right) \\
& \stackrel{(11)}{=} \frac{\left|S_{E}^{R}\right|}{2\left|S_{E}^{R}\right|-1} \mu_{i}\left(u_{R}\right)+\frac{\left|S_{E}^{R}\right|-1}{2\left|S_{E}^{R}\right|-1} \pi_{i}\left(u_{R}\right) .
\end{aligned}
$$

The value $\phi^{\widetilde{G}}$ is not necessarily a convex combination of the values $\mu$ and $\pi$ in communication situations for which the underlying game is not a unanimity game or the underlying communication network is not cycle-free.

Example 6 Let $N=\{1,2,3,4\}$ and let $E=\{\{1,2\},\{1,3\},\{2,4\},\{3,4\}\}$. The graph $(N, E)$ is depicted below.

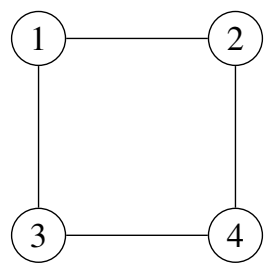

We have

$$
\begin{aligned}
\mathcal{N}_{E}^{\{1,2,3\}} & =\{\{1,2,3\}\} \\
\text { and } \mathcal{E}_{N}^{\{1,2,3\}} & =\{\{\{1,2\},\{1,3\}\},\{\{1,2\},\{2,4\},\{3,4\}\},\{\{1,3\},\{2,4\},\{3,4\}\}\} .
\end{aligned}
$$

We derive

$$
\begin{aligned}
w_{E}^{u_{\{1,2,3\}}} & =u_{\{1,2,3\}} \\
\text { and } \quad w_{N}^{u_{\{1,2,3\}}} & =u_{\{\{1,2\},\{1,3\}\}}+u_{\{\{1,2\},\{2,4\},\{3,4\}\}}+u_{\{\{1,3\},\{2,4\},\{3,4\}\}}-2 u_{E}
\end{aligned}
$$


and $w_{\widetilde{G}}^{u_{\{1,2,3\}}}=u_{\{1,2,3,\{1,2\},\{1,3\}\}}+u_{N \cup\{\{1,2\},\{2,4\},\{3,4\}\}}+u_{N \cup\{\{1,3\},\{2,4\},\{3,4\}\}}-2 u_{N \cup E}$.

Consequently,

$$
\begin{aligned}
\mu\left(u_{\{1,2,3\}}\right) & =\left(\frac{1}{3}, \frac{1}{3}, \frac{1}{3}, 0\right) \\
\text { and } \pi\left(u_{\{1,2,3\}}\right) & =\left(\frac{4}{12}, \frac{3}{12}, \frac{3}{12}, \frac{2}{12}\right) \\
\text { and } \phi^{\widetilde{G}}\left(u_{\{1,2,3\}}\right) & =\left(\frac{23}{70}, \frac{21}{70}, \frac{21}{70}, \frac{5}{70}\right) .
\end{aligned}
$$

Note that $\phi^{\widetilde{G}}\left(u_{\{1,2,3\}}\right)$ is not a convex combination of $\mu\left(u_{\{1,2,3\}}\right)$ and $\pi\left(u_{\{1,2,3\}}\right)$. $\Delta$

In general, a network control value is not necessarily a convex combination of the Myerson value and the position value, even if the underlying game is a unanimity game and the underlying communication network is cycle-free.

\section{Concluding remarks}

We conclude this paper with two examples of possible extensions of the decomposition theory to more general communication networks: undirected multigraphs and hypergraphs. For convenience, we restrict ourselves to an outline of the edge game and the corresponding position value in these examples.

Example 7 Let $\{1,2,3\}$ be the set of vertices and let $\{a, b, c, d\}$ be the set of edges of the multigraph depicted below, and consider the communication structure with underlying game $u_{\{1,3\}}$.

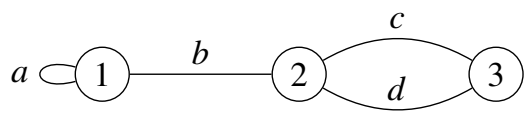

The collection of coalitions of edges which induce a minimal $\{1,3\}$-connecting edgeinduced subgraph is given by $\{\{b, c\},\{b, d\}\}$. The corresponding edge game can be written as $u_{\{b, c\}}+u_{\{b, d\}}-u_{\{b, c, d\}}$. The position value of this communication structure is given by $\left(\frac{2}{6}, \frac{3}{6}, \frac{1}{6}\right)$.

Example 8 Let $\{1,2,3,4\}$ be the set of vertices and let $\{\{1,2\},\{2,3\},\{2,3,4\}\}$ be the set of (hyper)edges of the hypergraph depicted below, and consider the communication structure with underlying game $u_{\{1,3\}}$. 


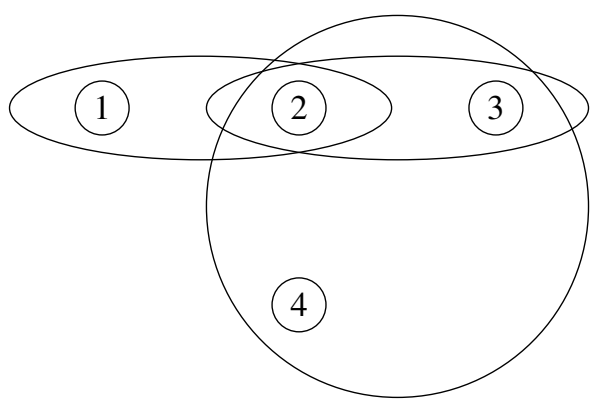

The collection of coalitions of edges which induce a minimal $\{1,3\}$-connecting edgeinduced subgraph is given by $\{\{\{1,2\},\{2,3\}\},\{\{1,2\},\{2,3,4\}\}\}$. The corresponding edge game can be written as $u_{\{\{1,2\},\{2,3\}\}}+u_{\{\{1,2\},\{2,3,4\}\}}-u_{\{\{1,2\},\{2,3\},\{2,3,4\}\}}$. The position value of this communication structure is given by $\left(\frac{12}{36}, \frac{17}{36}, \frac{5}{36}, \frac{2}{36}\right)$.

Hypergraph communication structures were introduced by Myerson (1980) and further studied by Van den Nouweland et al. (1992). Besides, Algaba et al. (2000) and Algaba et al. (2001) studied the position value and the Myerson value, respectively, for communication structures in which cooperation restrictions are modeled by union stable systems. Algaba et al. (2004) studied the relation between the position value for communication structures on hypergraphs and union stable systems. Future research could formalize these or other extensions to more general communication structures.

Open Access This article is distributed under the terms of the Creative Commons Attribution 4.0 International License (http://creativecommons.org/licenses/by/4.0/), which permits unrestricted use, distribution, and reproduction in any medium, provided you give appropriate credit to the original author(s) and the source, provide a link to the Creative Commons license, and indicate if changes were made.

\section{References}

Algaba E, Bilbao J, Borm P, López J (2000) The position value for union stable systems. Math Methods Oper Res 52(2):221-236

Algaba E, Bilbao J, Borm P, López J (2001) The Myerson value for union stable structures. Math Methods Oper Res 54(3):359-371

Algaba E, Bilbao J, López J (2004) The position value in communication structures. Math Methods Oper Res 59(3):465-477

Borm P, Owen G, Tijs S (1992) On the position value for communication situations. SIAM J Discret Math 5(3):305-320

Harsanyi J (1959) A bargaining model for the cooperative $n$-person game. In: Tucker A, Luce R (eds) Contributions to the theory of games IV. Princeton University Press, Princeton, pp 325-355

Myerson R (1977) Graphs and cooperation in games. Math Oper Res 2(3):225-229

Myerson R (1980) Conference structures and fair allocation rules. Int J Game Theory 9(3):169-182

Owen G (1986) Values of graph-restricted games. SIAM J Algebr Discret Methods 7(2):210-220

Shapley L (1953) A value for $n$-person games. In: Kuhn H, Tucker A (eds) Contributions to the theory of games II. Princeton University Press, Princeton, pp 307-317

van den Nouweland C, Borm P, Tijs S (1992) Allocation rules for hypergraph communication situations. Int J Game Theory 20(3):255-268

von Neumann J, Morgenstern O (1944) Theory of games and economic behavior. Princeton University Press, Princeton 